\title{
EXPERIÊNCIAS DE JOGOS DE MESA POR PESSOAS AUTISTAS
}

\author{
Fernando Emanoel de Oliveira Fernandes (discente)/UFPE \\ Laura Bezerra Martins (orientadora)/UFPE \\ Rafaella Asfora Siqueira Campos Lima (co-orientadora)/UFPE
}

\section{RESUMO}

A pesquisa, ainda em andamento, trata da experiência de jogo por adolescentes (entre 13 e 19 anos de idade) autistas verbais, residentes no Recife, com jogos de mesa. A problemática se desenvolve a partir da hipótese de que jogos de mesa podem impactar positivamente e estimular as habilidades sociais e comunicacionais dos indivíduos em questão, através das experiências de jogo. Devido às características sociocomunicativas de indivíduos com o transtorno do espectro autista (TEA) verbais, ou seja, com capacidade de se comunicar através da fala, estimular as habilidades de socialização e comunicação é, portanto, decisivo para que haja a possibilidade de engajamento social efetivo. Tais intervenções devem promover, além disso, a possibilidade de a pessoa autista traçar caminhos que reflitam em sua autonomia, sendo ele próprio agente de sua mudança, e bem-estar social, fazendo-o perceber os benefícios de uma vida em comunidade. Na busca por recursos alternativos que podem estimular positivamente as relações sociais, o raciocínio lógico, as habilidades sensoriais, criativas e lógicas, deparamo-nos com os jogos, em especial os jogos de mesa, os quais têm a propensão de ser capazes de instigar habilidades que venham a ampliar as possibilidades de acesso à independência e autonomia às pessoas com TEA verbais. Entende-se, também, que cada pessoa interpreta uma experiência em vários níveis diferentes e que as experiências e as emoções são capazes, inclusive, de mudar a forma como o sistema cognitivo opera. Então, ao unir o estudo dos jogos de mesa ao entendimento das experiências que estes artefatos lúdicos podem evocar nos sujeitos com TEA verbais, pretende-se, como objetivo geral da pesquisa, avaliar, como os elementos dos jogos de mesa impactam positivamente as habilidades comunicativas e sociais de jovens autistas verbais, visando o estímulo de atividades de integração social, através das percepções e do entendimento das experiências de jogo. Para tanto, serão utilizados o Método de Abordagem Indutivo buscando expandir a pesquisa do contexto micro para o macro, e os Métodos de Procedimento Experimental. Após definidas as metodologias, realizar-se-á um levantamento bibliográfico e uma revisão da literatura sobre os temas propostos e suas especificidades, a saber: TEA, jogos de mesa e experiência do usuário, com o objetivo de estabelecer embasamento teórico. Seguindo-se para a observação direta de como jovens autistas interagem com jogos de mesa; E, por fim, segue-se para a sistematização, a análise, o cruzamento e a comparação dos dados coletados.

Palavras-chave: Jogos de mesa; TEA; Experiência de jogo. 


\section{Introdução}

As políticas atuais de inclusão para pessoas com deficiências foram formatadas para garantir o pleno exercício do acesso, da permanência, da participação e aprendizagem da vida em sociedade de pessoas com deficiências (BRASIL, 2008). Conforme a Coordenadoria Nacional para Integração da Pessoa Portadora de Deficiência (CORDE), todos os valores básicos da igualdade de tratamento e oportunidade, da justiça social, do respeito à dignidade humana e bem-estar são garantidos e mais, justificados, pelas leis Federais.

Em 2015, entrou em vigor a Lei Brasileira de Inclusão da Pessoa com Deficiência (Lei no 13.146) (BRASIL, 2015). O Artigo 3 refere-se ao desenvolvimento do desenho universal como forma de acessibilidade, isso "[...] significa a concepção de produtos, ambientes, programas e serviços a serem usados, até onde for possível, por todas as pessoas, sem necessidade de adaptação ou projeto específico" (BRASIL, 2007).

Apesar da existência de recursos jurídicos que defendem uma sociedade mais justa e igualitária, o cenário desejado após a promulgação dessa Lei ainda não fora alcançado. Seu avanço tem sido pequeno e vagaroso, principalmente no tocante à inserção de pessoas com deficiências no convívio social.

Dados coletados por Zablotsky et al. em 2017, e por Christensen et al. em 2018, apontam para o crescimento de novos diagnósticos de jovens com transtorno do espectro autista (TEA) no mundo, pois à medida que ocorrem avanços científicos sobre o TEA, mais se conhece sobre suas diferentes manifestações. Isso leva a melhores condições de deteç̧ão da condição em um indivíduo. Tais sujeitos possuem capacidade de interagir através da fala, mas com dificuldade, além de problemas nas habilidades de socialização e comunicação.

$\mathrm{Na}$ busca por recursos alternativos e artefatos culturais que possam incluir socialmente pessoas autistas e promover o desenvolvimento e a aprendizagem, de maneira diferenciada, estimulando das relações sociais, o raciocínio lógico, as habilidades sensoriais, criativas e lógicas, deparamo-nos com os jogos. Dentre eles, jogos de mesa, os quais podem instigar habilidades que venham a ampliar as possibilidades de acesso à independência e autonomia às pessoas com TEA (BEN-SASSON et al. 2014). Tais artefatos são constituídos por elementos concretos (dados, cartas, peças, fichas...) e por princípios básicos que, apesar de sua função primordial ser a do entretenimento (HAYSE 2018, p.289), têm grande impacto na intercomunicação e, podem proporcionar experiências únicas, devido às dinâmicas interativas entre os participantes. 
É importante entender, também, que cada pessoa interpreta uma experiência em níveis diferentes, sendo eles: a) o visceral: aparências, às primeiras percepções, aos sentidos; b) o comportamental: às sensações se têm ao utilizar tal artefato; e, c) o reflexivo: ao raciocínio, às interpretações e compreensões sobre tal objeto (NORMAN 2008, p.56-58). Cada um desses níveis atua para gerar experiências para os indivíduos.

Ao unir o estudo dos jogos de mesa ao entendimento das experiências que estes artefatos lúdicos podem evocar nos sujeitos com TEA, pretende-se avaliar como os elementos dos jogos de mesa impactam as habilidades sensoriais, comunicativas e sociais de jovens autistas verbais com dificuldade de comunicação, visando o estímulo de atividades de socialização, das aptidões sensoriais, da criatividade e da memorização, através das percepções e do entendimento das experiências de jogo.

\section{Fundamentação Teórica}

O afastamento social de pessoas com deficiências é considerado hoje como uma injúria à dignidade humana. O ser humano, sem exclusões, deve ser colocado à condição de respeitabilidade e, apesar das limitações, não devem ser vistos como inferiores ou incapazes (BRASIL, 2015, p.379). Logo, nenhuma pessoa que possui quaisquer deficiências deverá ser posta à margem da sociedade e dos benefícios e direitos que esta proporciona aos indivíduos viventes numa comunidade, como o direito primordial à vida.

Apesar dos esforços pela diminuição da exclusão social, o preconceito e a ignorância ainda fazem parte do cotidiano nacional. Pessoas com autismo, também conhecido como transtorno do espectro autista (TEA), um transtorno do neurodesenvolvimento que se manifesta de diversas condições e múltiplas especificações clínicas individuais (APA, 2014, p. 03), sofrem, sobretudo, com o preconceito e a falta de conhecimento da sociedade.

O indivíduo com autismo é caracterizado, permanentemente, com prejuízo na interação social, déficits comportamentais qualitativos na comunicação, padrões limitados ou estereotipados de comportamentos, repertório restrito de interesses e atividades, onde as anormalidades nestes pontos surgem por volta dos três anos de idade. (APA, 2014, p. 03). 
Tratando-se de adolescentes com TEA, pesquisas relataram maior incidência em prejuízos na comunicação, tanto verbal, quanto não verbal, bem como a diminuição de repetições no comportamento e ampliação das dificuldades de reciprocidade social. Vale salientar que, a fase da adolescência para indivíduos com TEA, pode vir a ser mais complicada que em pessoas consideradas típicas, ocorrendo diversas situações de extrema conturbação para os sujeitos.

Dentro desse espectro há variações de níveis e estados pelos quais os indivíduos com TEA estão sujeitos. Estas caraterísticas, de forma alguma, podem ser utilizadas para uniformizar tais sujeitos dentro de um grupo homogêneo. Por encontrar-se em um espectro, o autismo demonstra uma ampla diversidade de especificidades e níveis de comprometimento, desde o mais leve ao mais grave. Há pessoas que não são tipicamente verbais, afastadas de qualquer interação social e, em outro nível mais brando, há aqueles indivíduos que podem aceitar interações sociais, conseguindo se relacionar socialmente, porém não o fazendo da forma típica, possuindo dificuldades em regular a interação social após iniciada (APA, 2014, p. 03).

Neste grau mais brando do TEA, que servirá como objeto de estudo para este trabalho, apesar de possuírem as dificuldades presentes no autismo, aparecem em grau menor de comprometimento e, geralmente, as pessoas possuem alta fixação em uma área que lhes despertem interesse. Em sujeitos neste espectro do transtorno, a capacidade de interesse social pode vir a crescer com o passar do tempo. Com a progressão do desenvolvimento, atividades em que haja interações com pessoas, em quaisquer esferas sociais, tornam-se aceitáveis e até oportunas para os autistas (GRACIOLI; BIANCHI, 2014, p.128).

A inclusão de pessoas autistas deve ser associada a diferentes intervenções para que o indivíduo em questão possa ter autonomia na sua vivência em sociedade, e propiciar, ainda, seu bem-estar e de suas relações, libertando-o de seu universo segregado, notando as benfeitorias de se criar vínculos com outras pessoas presentes ao seu redor (GRACIOLI; BIANCHI, 2014, p.129).

A inclusão de pessoas com deficiências ainda é um desafio para desenvolvedores e designers de jogos de mesa. Mesmo nos principais mercados de jogos, ainda não há um olhar atencioso para práticas acessíveis, especialmente quando se observa os de jogos de mesa. Isso porque, desenvolver artefatos acessíveis possui alto custo de produção e complexidade (BARLET e SPOHN, p. 8, 2012). 
Entendemos que seja necessário considerar uma forma alternativa de abordagem para a inclusão de pessoas, considerando não apenas o jogo, mas também, a ambientação propícia para o acesso das pessoas aos jogos. Ou seja, não basta um jogo ser acessível se a indústria não fomenta e cria oportunidades para que as pessoas tenham conhecimento e acesso a esses artefatos lúdicos (BARLET e SPOHN, p. 9, 2012).

Lieberman e Houston-Wilson (2009, p. 64), indicam quatro adaptações necessárias para que seja possível desenvolver estratégias de inclusão no desenvolvimento de jogos: modificação nos equipamentos, nas regras, no ambiente e na instrução. Além disso, quando pensamos em experiência de jogo por pessoas com espectro do autismo, especialmente as do recorte tratado aqui, deve-se pensar que há diversos agentes envolvidos - além dos jogadores, a família e a sociedade - os quais, em sintonia, contribuem para que o processo de inclusão seja eficaz.

Para que a experiência do jogador autista seja satisfatória para ele, é necessário entender as expectativas desses indivíduos, devendo o jogo se adaptar ao jogador, às suas necessidades, dificuldades e potencialidades. Em outras palavras, o jogador não terá uma experiência positiva se tal artefato não oferecer condições de o indivíduo se sentir plenamente capaz de usufruir, de forma autônoma, e explorar todas as potencialidades do jogo.

À vista disso, como uma experiência pode ser vivenciada em níveis distintos, e, ao jogar, o indivíduo adquire e estimula uma vasta gama de habilidades e conhecimentos, o estudo das experiências e a forma como elas impactam os jogadores autistas necessita de uma maior compreensão para que profissionais que atuam no marcado de jogos possam criar artefatos lúdicos capazes de satisfazer os anseios e expectativas desses jogadores (NORMAN 2008, p.64).

Neste âmbito, o grande desafio dos designers de jogos é o de encontrar formas inovadoras de adaptar os conteúdos lúdicos às necessidades dos jogadores autistas, flexibilizando assuntos, apresentando novos significados às experiências de jogo, de forma que todos os jogadores, autistas ou não, sejam capazes de jogar não ferindo a função primordial da inclusão social que é a de abolir a discriminação e o isolamento social das diferenças (BARLET e SPOHN, p. 40, 2012). 
Como forma de buscar experiências imersivas e inclusivas para pessoas no espectro do autismo verbal, foi observado que elementos e mecanismos capazes de favorecer e estimular habilidades sensoriais, sociais, capacidade de memorização, além de reconhecimentos visuais e espaciais podem ser de grande valia para ofertar tais necessidades (BEN-SASSON et al. 2014). Na procura por possíveis soluções que atendessem às demandas de estímulos, lançou-se a hipótese de se utilizar jogos analógicos como forma de produzir experiências de jogo positivas para as interações comunicacionais e sociais de pessoas autistas verbais.

Jogos analógicos são utilizados devido à sua ludicidade e à capacidade de interação social em tempo real constantes. Além do mais, as experiências que tais jogos oferecem, são fundamentais para o desenvolvimento de sujeitos com transtorno no espectro autista. Este modelo de jogo, que promove a interação direta (seja por oposição ou por cooperação) entre jogadores, possui ancestrais comuns, como Senet, Mancala, Pachisi e Mehen (SALEN e ZIMMERMAN 2004). Norman, em seu livro "Design Emocional", comenta que as experiências e as emoções são capazes, inclusive, de mudar a forma como o sistema cognitivo opera (NORMAN 2008, p.38).

O objetivo do jogo de mesa como técnica de estímulo das habilidades de socialização e comunicação deve ser simples e motivador, com linguagem compreensível pelos usuários, com o ritmo dado pelo grupo que joga, sem ser massificado e que seja criado para apoiar o desenvolvimento sócio comunicacional do indivíduo (ROSA et al. 2004). Rizzo (2001) menciona que as características de ludicidade, a interação entre os jogadores, o estímulo das relações e o convívio social de sujeitos com perfis diferenciados com o propósito do divertimento, apresentam resultados satisfatórios e significativos para a sociabilidade dos indivíduos autistas.

Além do caráter lúdico, o potencial dos jogos caminha desde entendê-lo como uma ferramenta auxiliar para profissionais da área da saúde e da educação, através do estímulo e da aprendizagem tangencial das habilidades sensoriais, sociais e comunicacionais, onde o objetivo principal não se encontra no conteúdo (PRENSKI 2012), até a serem jogos com a função propriamente de transmitir determinado conteúdo, os chamados edutainment (EGENFELDT-NIELSEN et al. 2008). 
O jogo se mostra como uma atividade interativa e, se bem desenvolvida, pode gerar experiências diversas e positivas, capazes de estimular habilidades sensoriais, criativas e lógicas (BUSARELLO, BIEGING e ULBRICHT, 2015, p.246). Os jogos expandem a experiência do jogador, potencializam o raciocínio e as tomadas de decisões, aumentam o leque de ações e são capazes de melhorar as capacidades de sensoriais, o engajamento, o contato e a interação social, fundamentais para o desenvolvimento humano. Ao vislumbrar a atuação dos jogos e suas experiências proporcionadas, buscou-se formas de construir uma posição em relação à diversão e ao desenvolvimento social. Atrelar a ludicidade a temáticas complexas e abstratas é um diferencial para a amplificação das experiências de jogo positivas de pessoas autistas, além de promotora de interações sociais.

Surgiu então, a hipótese de que experiências de jogo positivas proporcionadas por jogos de mesa são capazes de estimular as habilidades comunicativas e de interação social de jovens com espectro de autismo verbal.

\section{Trajetória Metodológica do Projeto}

Tratando-se de uma análise do impacto que experiências de jogo têm sobre pessoas com transtorno do espectro autista, a priori será utilizado o Método de Abordagem Indutivo, partindo-se da observação de experiências com alguns jogos de mesa por jovens autistas, pretende-se expandir as informações obtidas para generalizá-las.

Como Métodos de Procedimento, serão utilizados o Método Experimental, selecionando as variáveis capazes de influenciar as experiências positivas proporcionadas pelos jogos de mesa, definindo as formas de controle e de observação dos efeitos que as variáveis produzem nos jogadores, com a finalidade de observar as interações e as experiências entre os jovens autistas e os jogos de mesa. $O$ Método Funcionalista servirá para analisar as experiências do ponto de vista da função de cada elemento dos jogos de mesa.

Após o levantamento bibliográfico e de revisão da literatura sobre os temas, a pesquisa seguirá para a análise morfológica dos jogos de mesa previamente selecionados com base na Metodologia de Design de Jogos de Flanagam e Nissenbaum (2016), vislumbrando encontrar elementos em comum e, às observações diretas de como jovens autistas verbais interagem com jogos de mesa. 
Seguindo-se para a análise, para o cruzamento e para a comparação dos dados coletados, e então, estudá-los a partir de um feixe analítico priorizando as relações entre jogos de mesa, pessoas com espectro de autismo verbal e experiência de jogo, a fim de se avaliar a ocorrência de alterações significativas nas habilidades sensoriais, comunicacionais e sociais, e quais impactos dessas mudanças nesses sujeitos.

Essas etapas são úteis para pontuar como as conexões entre determinados elementos, a exemplo das grandes áreas abordadas (jogos de mesa, experiência do usuário e psicologia), possibilitam arranjos que têm por consequência uma nova dinâmica no modo como os sujeitos estão se ligando, isto é, altera-se as formas assumidas numa relação.

\section{Considerações Parciais do Projeto}

Até o momento, a presente pesquisa, ainda em andamento, já se debruçou sobre as temáticas dos jogos de mesa, do público de pessoas com TEA, além da temática de experiência do usuário, esmiuçando características e pormenores que permeiam os assuntos.

Como resultado parcial, foram identificados quais elementos nos jogos de mesa são capazes de proporcionar experiências aos jogadores, restando o entendimento de como esses elementos estimulam tais experiências. Percebeu-se ainda, por meio de uma revisão sistemática da literatura, que pesquisas envolvendo jovens com TEA ainda surgem em número bastante reduzido, tornando-se uma oportunidade para ampliar o leque de pesquisas existentes, mostrando a relevância do presente trabalho. No âmbito da temática da experiência, foi possível compreender os diferentes níveis de interpretação das sensações e emoções advindas das experiências e indivíduos com produtos, propostos por Jordan (2002), Desmet (2002), Norman (2008) e Damásio (2011).

Os esforços estarão, a partir de agora, destinados a conectar as três dimensões temáticas do projeto e, através de ferramentas como entrevistas e observações, elucidar questões ainda pertinentes.

\section{Referências}

APA - American Psychiatric Association. Manual Diagnóstico e Estatístico de Transtornos Mentais DSM-5. Tradução de Maria Inês Corrêa Nascimento et al. Porto Alegre: Artmed, 2014.

BARLET, Steven e SPOHN, Steve. Includification: A Pratical Guide to Game Acessibility. Ablegamers Fuoundation, 2012.

BEN-SASSON, Ayelet; LAMASH, Liron; GAL, Eynat. To Enforce or not to Enforce? The Use of Collaborative Interfaces to Promote Social Skills in Children with High Functioning Autism Spectrum Disorder. SAGE Publications. Texas, EUA, 2014. 
BRASIL. Convenção sobre os Direitos das Pessoas com Deficiência. Protocolo Facultativo à Convenção sobre os Direitos das Pessoas com Deficiência. Sistema Nacional de Informações sobre Deficiência SICORDE. Brasília, 2007. Disponível em:

$<$ http://portal.mec.gov.br/index.php?option=com_docman\&view=download\&alias=424-cartilhac\&category_slug=documentos-pdf\&ltemid=30192>.

BRASIL. Acessibilidade: Legislação Federal. Secretaria Especial dos Direitos Humanos. Coordenadoria Nacional para a Integração da Pessoa Portadora de Deficiência, CORDE. Brasília, DF, 2008.

BRASIL. Lei no 13.146, de 6 de julho de 2015. Institui a Lei Brasileira de Inclusão da Pessoa com Deficiência (Estatuto da Pessoa com Deficiência). Disponível em:

<http://www.planalto.gov.br/ccivil_03/_Ato2015-2018/2015/Lei/L13146.htm\%3E.>. Acesso em: 21 de fevereiro de 2020.

BUSARELLO, Raul Inácio, BIEGING, Patrícia, ULBRICHT, Vania Ribas. Inovação em Práticas e Tecnologias para Aprendizagem. São Paulo, 2015.

CHRISTENSEN, D. L., BRAUN, K., BAIO, J., BILDER, D., CHARLES, J., CONSTANTINO, J. N., DANIELS, J., DURKIN, M. S., FITZGERALD, R. T., KURZIUS-SPENCER, M., LEE, L. C., PETTYGROVE, S., ROBINSON, C., SCHULZ, E., WELLS, C., WINGATE, M. S., ZAHORODNY, W., \& YEARGIN-ALLSOPP, M. Prevalence and Characteristics of Autism Spectrum Disorder among Children Aged 8 Years-Autism and Developmental Disabilities Monitoring Network, 11 Sites, United States 2012. Morbidity and Mortality Weekly Report. Surveillance summaries (Washington D.C.: 2002), 65 (13), 1-23, 2018.

DAMÁSIO, A. R. O Erro de Descartes: Emoção, Razão e o Cérebro Humano. Temas e Debates, 2011.

DESMET, P. Designing Emotions. Delft: Delft University of Technology, 2002.

EGENFELDT-NIELSEN, S. et. al. Understanding Videogames: The Essential Introduction. Londres: Editora Routledge, 2008.

FLANAGAN, Mary e NISSENBAUM, Helen. Value at Play: Valores em Jogos Digitais. São Paulo: Blucher, 2016.

GRACIOLI, Maria Madalena; BIANCHI, Rafaela Cristina. Educação do Autista no Ensino Regular: Um Desafio à Prática Pedagógica. Nucleus, v.11, no 2, Outubro 2014.

HAYSE, Mark. Tabletop Games and 21st Century Skill Practice in the Undergraduate Classroom. MidAmerica Nazarene University, 2018.

JORDAN, P. The Personalities of Products. Pleasure With Products: Beyond Usability. London: Taylor and Francis, 2002.

LIEBERMAN, L. J.; HOUSTON - WILSON, C. Strategies for Inclusion: A Handbook for Physical Educators. 2.ed. Champaign: Human Kinetics, 2009.

MCLUHAN, Marshall. Os Meios de Comunicação como Extensões do Homem. São Paulo: Cultrix, 2007.

NORMAN, Donald A. Design Emocional: Por que Adoramos (ou Detestamos) os Objetos do dia a dia. Rio de Janeiro; Rocco, 2008.

RIZZO, Gilda. Jogos Inteligentes: A Construção do Raciocínio na Escola. Ed. Bertrand Brasil, Rio de Janeiro, 2001;

ROSA, R. S. D.; BENEVIDES, R. E.; MACIEL, J. B.; MONTEIRO D.; BERNARDES, R. M. Recursos Didáticospedagógicos na Promoção da Educação Popular em Saúde. Anais do 2을 Congresso Brasileiro de 
Extensão Universitária. Belo Horizonte. Minas Gerais. 2004. [Internet]. [citado 2016 Jan 20].

Disponível em: <https://www.ufmg.br/congrext/Saude/Saude176.pdf>. Acesso em 18 de fevereiro de 2020.

SALEN, Katie; ZIMMERMAN, Eric. Rules of Play - Game Design Fundamentals. Londres: Editora The MIT Press Cambridge, 2004.

ZABLOTSKY, B., BLACK, L. I., \& BLUMBERG, S. J. Estimated Prevalence of Children with Diagnosed Developmental Disabilities in the United States, 2014-2016. NCHS Data Brief, 291, 1-8, 2017. 